\title{
Numerical Simulation of Blood Flow in Double-Barreled Cannon EVAR and its Clinical Validation
}

\author{
Rui-Hung Kao ${ }^{1 \#}$, Wei-Ling Chen ${ }^{2 \#}$, Tzong-Shyng Leu ${ }^{1}$, Tainsong Chen ${ }^{2}$ and Chung-Dann Kan ${ }^{3 *}$ \\ ${ }^{1}$ Department of Aeronautics and Astronautics, National Cheng Kung University, Tainan, Taiwan \\ ${ }^{2}$ Department of Biomedical Engineering, National Cheng Kung University, Tainan, Taiwan \\ ${ }^{3}$ Department of Surgery, National Cheng Kung University Hospital, Tainan, Taiwan \\ \#These authors contributed equally to this work and should be considered co-first authors
}

\begin{abstract}
The Double-Barreled cannon stent-graft Aortic Repair (DoBAR) strategy is a specific method for fixing extremely dilated aortic landing-zone issue. Its clinical validation and application may be examined using computational schemes. Three assumed conditions: the single stent-graft, the longitudinal direction (LD)-type DoBAR, and the sagittal direction (SD)-type DoBAR models were examined by computational fluid dynamics (CFD) simulations. Two specific points in one cardiac cycle were plotted. The non-dimensional frequency parameters were calculated to evaluate the flow field stabilities. The primary axial flow skewed from inner to outer aortic portion after passing through the aortic arch at peak forward-flow time (PFFT) and formed the swing signature at peak reverse-flow time (PRFT). The secondary flow developed as counter-rotating vortices of this model. In LD-type, the septum of anteroposterior chambers separated the primary flow to form two individual axial flows, the morphology resembled as the single model at PFFT. In SD-type, the septum of outer-inner chambers divided the flow pathway into two layers, and then weakened the reversed flow strength at PRFT. The septum of the DoBAR limited the development of secondary flow and the swing pattern disappeared in both types. The Strouhal and Wormersly numbers showed the flow pulsatility intensity decreased with steadier flow in both DoBAR models. The Dean numbers disclose the SD-type had weaker axial velocity and weaker secondary flow.
\end{abstract}

Conclusion: CFD models might help us observe and understand the hemodynamic and blood flow field changes and the potential effects caused by the application of the DoBAR strategy.

Keywords: Aortic aneurysm; Computational fluid dynamics; Thoracic endovascular aneurysm repair; Axial velocity distribution; Secondary flow; Double-barreled cannon stent-graft aortic repair (Dobar)

\section{Introduction}

Constant high blood pressure can cause damage in the aortic intimal layer and result in a weakened arterial wall. The weakened arterial wall can then enlarge and form a bulge sac. When the aortic diameter is more than 1.5 times the normal aortic diameter, it is referred to as an aortic aneurysm. Aneurysms can form in any artery throughout the body. The descending aorta and the infra-renal aorta are two of the most affected structures. Aortic aneurysms can rupture and cause life-threatening internal bleeding. The incidence of a thoracic aortic aneurysm is approximately 5-10 per 100000 patient years, at least 3\%$4 \%$ of which occurs in patients aged more than 65 years old $[1,2]$.

Traditional open surgery with graft interposition is the goldstandard strategy for aneurysm disease, but it is associated with high mortality and morbidity in older patients [3]. Thoracic endovascular aneurysm repair (TEVAR) surgery significantly reduces perioperative mortality and morbidity. TEVAR has thus become the main alternative method for older patients. Although significant advances have been made in industrial and technological research, the extremely dilated aortic landing zone remains the biggest obstacle to a successful surgery because of the limited availability of graft sizes. We began developing the novel double-barreled cannon stent-graft aortic repair (DoBA R) strategy as a life-saving technique for patients with enlarged aortic landing zones [4]. However, the feasibility of the DoBAR strategy still requires further examination.

After originating from the heart, the aorta bends to form the aortic arch, turning more than $180^{\circ}$ to become the descending and abdominal aorta. In addition to anterograde pulsatile flow, blood flow through the aortic arch may develop into swirling secondary flow because of centrifugal force. The secondary flow exhibits a velocity field that skews toward the outer wall of the arch [5]. In this study, we studied the secondary flow from the aortic arch to the descending aorta, using computational fluid dynamics (CFD) techniques. The non-dimensional frequency parameters of Womersley numbers, Strouhal numbers, and Dean numbers were used to evaluate the blood flow field.

\section{Material and Methods}

This study uses CFD numerical simulation to determine the feasibility of using the DoBAR method to examine the extremely dilated landing zone of aortic aneurysms. Figure 1 shows the three representative stent-graft models analyzed in this study. The first model analyzed was the single stent-graft model, which is the model typically used to mimic the treatment of thoracic aortic aneurysms by TEVAR (Figure 1A). The second model analyzed was the longitudinal directiontype (the sagittal direction $0^{\circ}, \mathrm{LD}$ ) DoBAR model placed inside the aorta at $0^{\circ}$ to the sagittal plane (Figure 1B). The third model was the

*Corresponding author: Chung-Dann Kan, Department of Surgery, National Cheng Kung University Hospital, Tainan, Taiwan, Tel: +886-6-235-3535 ext 3497; E-mail:kcd56@mail.ncku.edu.tw

Received July 14, 2014; Accepted October 17, 2014; Published October 20 2014

Citation: Kao RH, Chen WL, Leu TS, Chen T, Kan CD (2014) Numerical Simulation of Blood Flow in Double-Barreled Cannon EVAR and its Clinical Validation. J Vasc Med Surg 2: 160. doi: 10.4172/2329-6925.1000160

Copyright: $\odot 2014 \mathrm{Kao}$ RH, et al. This is an open-access article distributed unde the terms of the Creative Commons Attribution License, which permits unrestricted use, distribution, and reproduction in any medium, provided the original author and source are credited. 


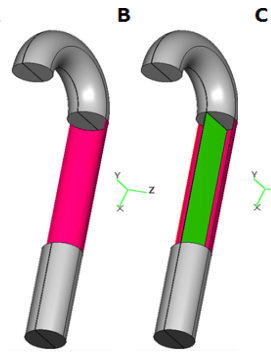

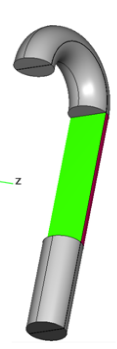

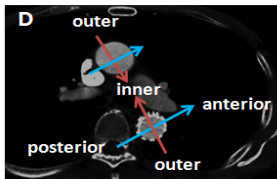

E LD type

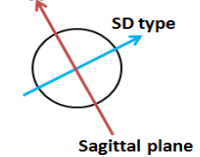

Figure 1: The models of the aortic stent grafts. (A) Single stent-graft, (B) the longitudinal direction (LD) type of DoBAR, (C) the sagittal direction (SD) type of DoBAR, (D) the direction of the descending aorta, $(E)$ The conditions of LD and SD type in the descending aorta.
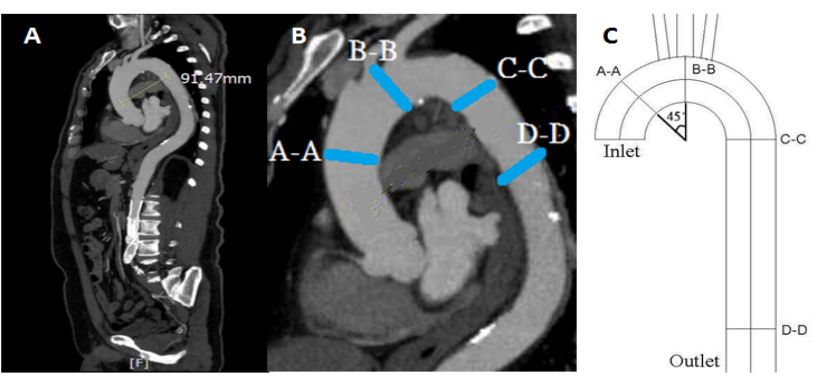

Figure 2: $(A)$ the real geometry of the thoracic aorta based on the medical computed tomography image. (B), (C) the definition of the four sampling planes.

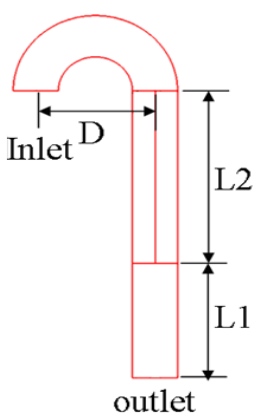

Figure 3: (A) The simplified geometry model of the thoracic aorta: the diameter of curvature of the aortic arch $D=95 \mathrm{~mm}$, the inside lumen diameter $\mathrm{D} 1=36 \mathrm{~mm}$, the thickness of the stent graft was $0.2 \mathrm{~mm}$ and the double stent graft $G=0.4 \mathrm{~mm}$, the length of the stent $L 2=150 \mathrm{~mm}$, the length of the downstream aorta $L 1=100 \mathrm{~mm}$; $(B)$ the LD type of DoBAR; (C) the SD type of DoBAR.

sagittal direction-type (the sagittal direction $90^{\circ}$, SD) DoBAR model, which we placed inside the aorta at $90^{\circ}$ to the sagittal plane (Figure 1C). The orientation of the anterior, posterior, inner, outer, and sagittal planes and LD and SD types are displayed in Figure 1D and 1E. The figures show that the DoBAR consists of two stent-grafts that compete for space within the aorta. To obtain the best balanced compliance, two equally sized stent-grafts were chosen. If both endovascular stent-grafts expanded to their maximum size, the girth of each stent-graft would require to fit the diameter and half of the circumference. Therefore, these two stent-grafts would form two semicircles [4]. To comprehensively understand the changes in the flow fields after inserting the proposed DoBAR into the aorta, blood flow fields for both the longitudinal and sagittal double stent-grafts inside the dilated aorta need to be studied. The CFD analysis in this study involves three aspects: the construction of a 3D geometrical model and mesh generation, a definition of boundary conditions, and a subsequent numerical calculation.

\section{The aortic CFD geometry}

Figure $2 \mathrm{~A}$ and $2 \mathrm{~B}$ show the real geometry of the thoracic aorta based on the medical computed tomography (CT) examination image. The CT scan covers the entire target region with a resolution of $1024 \mathrm{x}$ 768 pixels and a slice thickness of $5 \mathrm{~mm}$. Figure 2C shows the simplified geometric model of the CT image by ESI CFD-RC GEOM (Alabama, USA) for the surface and grid generation. To achieve grid independence, we created more than 500000 elements in the fluid domain for each model. We assessed flow fields at four cross-planes: the ascending aorta (A-A plane), the mid-aortic arch (B-B plane), the stent-graft proximal landing zone (C-C plane), and the stent-graft distal landing zone (D-D plane), as shown in Figure 2B and 2C. Figure 3 shows the dimensions of the model: the diameter of curvature of the aortic arch $\mathrm{D}$ was 95 $\mathrm{mm}$, the inside lumen diameter D1 was $36 \mathrm{~mm}$, the thickness of the stent-graft was $0.2 \mathrm{~mm}$, the thickness of the double stent-graft $\mathrm{G}$ was $0.4 \mathrm{~mm}$, the length of the stent $\mathrm{L} 2$ was $150 \mathrm{~mm}$, and the length of the downstream stent L1 was $100 \mathrm{~mm}$. Figure 3B shows the LD type of the DoBAR model, and Figure 3C shows the SD type.

The 3D unsteady continuity equation and the Navier-Stokes equation, as shown below, were used to solve the numerical solution for the blood flow field, using CFD-ACE plus.

$$
\begin{aligned}
& \frac{\partial \rho}{\partial t}+\nabla \bullet(\rho \vec{V})=0 \\
& \frac{\partial \vec{V}}{\partial t}+\vec{\nabla} \bullet(\vec{V} \vec{V})=-\frac{1}{\rho} \vec{\nabla} p+\frac{1}{\operatorname{Re}} \nabla^{2} \vec{V}
\end{aligned}
$$

where $\rho$ is the fluid density, $\vec{V}$ is the velocity vector of the flow field, $\mathrm{Re}$ is the Reynolds number, and $p$ is pressure.

\section{Boundary conditions}

This study models the rheological properties of aortic blood as

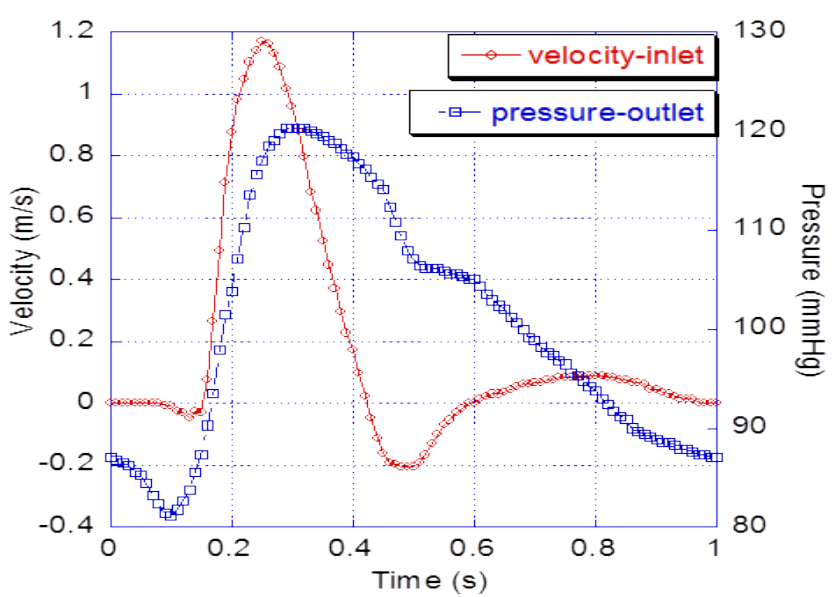

Figure 4: Blood velocity and pressure waveform of a normal adult at $1 \mathrm{~Hz}$. 
an incompressible, homogeneous, and Newtonian fluid [6-8]. Blood flows in the vessels are pulsatile. Figure 4 shows the physiologically representative pulsatile velocity-time sequence at the inlet and the pressure waveform at the outlet for typical normal adult conditions [6,7]. The highest blood flow speed is achieved during the systole, and the lowest speed is followed by the diastole. The standard for determining the laminar or turbulent flow of a fluid is the Re number. At $\operatorname{Re}<2300$, the fluid is commonly regarded as laminar. Previous research has treated the blood flow in large blood vessels as laminar [6]. The mean Re number in current flow conditions was approximately 2000. Therefore, the flow simulations in this study assume a laminar flow. To obtain a periodic state in a time-dependent calculation in CFD simulations, this study adopts the semi-implicit method for pressurelinked equations consistent (SIMPLEC). This study simulated four cardiac cycles to achieve a periodically convergent solution independent of the initial conditions. In total, 100 time steps were used to resolve one cycle. We defined the hemodynamic fluid parameters as follows: blood viscosity of $0.0035 \mathrm{Ns} / \mathrm{m}^{-2}$ and blood density of $1050 \mathrm{~kg} / \mathrm{m}^{-3}$ [6]. This study applied the no-slip boundary condition on all vessel walls, and regarded them as rigid walls.

\section{Secondary flow and dimensionless number for pulsating flow}

The aorta has a complex $3 \mathrm{D}$ U-shaped geometry with a $180^{\circ}$ arch. When a large volume of blood passes through the curved aortic arch, the blood fluid is subjected to a centrifugal acceleration normal to the original flow direction. The centrifugal force causes $v$ and $w$ velocity components in addition to axial flow velocity $\mathrm{u}$. The moving blood forms a secondary flow pattern and develops into two counter-rotating vortices $[9,5]$. The strength $(Q)$ of the secondary flow can be evaluated using (3):

$$
Q=\sqrt{v^{2}+w^{2}}
$$

where $v$ and $w$ are the velocity components in the $\mathrm{y}$ and $\mathrm{z}$ directions, respectively.
A dimensional analysis of the unsteady U-shaped pipe flow commonly leads to non-dimensional values for Re, for the Womersley number $(\alpha)$, for the Strouhal number (St), and for the Dean number (De) [5,9-11]. This study defined these parameters in (4) to (7), and used them to characterize the current unsteady blood vessel flow. The Reynolds number Re is defined as the ratio between the inertial force and the viscous force. It can be used to determine if flow changes from laminar flow to turbulent flow. The Womersley number represents the ratio of unsteady forces to viscous forces, and the Strouhal number represents the ratio of the oscillatory inertial forces to the convective inertial forces. When the Womersley number is larger than 10 , the flow tends to be unstable [5]. A low Strouhal number indicates higher pulsatility [8]. The Dean number, defined as the ratio of centrifugal forces to viscous forces $[5,11]$, indicates the strength of secondary flow.

$$
\begin{aligned}
& \operatorname{Re}_{D 1}=\frac{\rho V_{m} D}{\mu} \\
& \alpha=\frac{D}{2} \sqrt{\frac{2 \cdot \pi \cdot f \cdot \rho}{\mu}} \\
& S t=\frac{D}{2} \cdot \frac{f}{V_{p}-V_{m}} \\
& D e=\frac{\mu D_{1}}{v} \sqrt{\frac{D_{1}}{2 D}}
\end{aligned}
$$

where $\rho$ is the fluid density, $\mathrm{V}_{\mathrm{m}}$ is the mean axial velocity at a specific cross-section, $\mathrm{D}$ is the aortic diameter, $\mu$ is the dynamic viscosity of the blood, $\mathrm{f}$ is the heart rate, $\mathrm{V}_{\mathrm{p}}-\mathrm{V}_{\mathrm{m}}$ is the difference between the peak and mean velocity at the cross-section, $\mathrm{D}_{1}$ is the tube diameter, is the kinematic viscosity, and $\mu$ is the velocity component in the $\mathrm{x}$ direction.

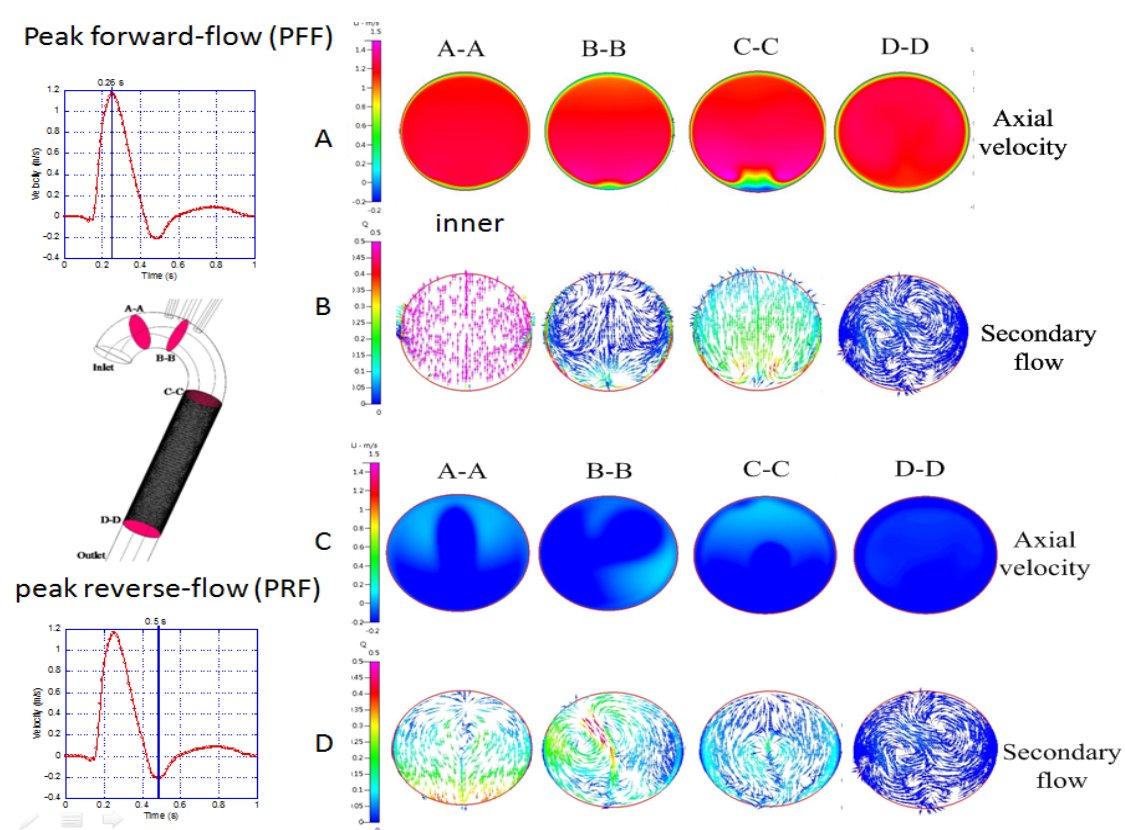

Figure 5: The CFD results of the single stent-graft model. (A) Axial velocity on $P F F(T=0.26 s)$, (B) secondary flow on $P F F$, (C) axial velocity on $P R F(T=0.5 s)$, (D) secondary flow on PRF. 


\section{Results}

\section{Primary axial velocity and secondary flow distribution}

According to the pulsatile inlet velocity boundary condition (Figure 4 ), this study plotted two specific time points during one cardiac cycle. The first time plotted was the peak forward-flow time (PFFT) at T = $0.26 \mathrm{~s}$, and the second was the peak reverse-flow time (PRFT) at $\mathrm{T}=$ $0.5 \mathrm{~s}$. This study plotted flow fields at four cross-planes: the A-A, B-B, $\mathrm{C}-\mathrm{C}$, and D-D planes along the simplified aorta model, as shown in Figure 2C.

\section{The single stent-graft model}

The primary axial velocity and secondary flow fields for the single stent-graft model are shown in Figure 5. At the PFFT point of the systole, the axial velocity distribution was uniform, with an average velocity of approximately $1.2 \mathrm{~m} / \mathrm{s}$ at the ascending aorta (Figure $5 \mathrm{~A}$, A-A plane). When the flow passed through the U-shaped aortic arch, the axial flow velocity skewed and accelerated near the inner region of the U-shaped aortic arch from the arch to the proximal descending aorta (Figure 5A, B-B and C-C planes). The peak axial velocity reached $1.4 \mathrm{~m} / \mathrm{s}$. Near the outer region of the U-shaped aortic arch, the axial velocity distribution remained uniform at approximately $1.2 \mathrm{~m} / \mathrm{s}$. The findings also showed that the axial velocity close to the inner region of the U-shaped aortic arch decelerated significantly (Figure 5A, C-C plane). After entering the distal descending aorta, the primary axial flow velocity recovered to near that of the inlet flow (Figure 5A, D-D plane). Because of the centrifugal forces present, the flow around the curvature of the aortic arch was expected to induce secondary flow. The magnitudes of the secondary flow velocity $(\mathrm{Q})$ were plotted with a color bar, and the secondary flow structures were indicated with velocity vectors. At the ascending aorta A-A plane, the axial velocity was uniform, and the secondary flow was beginning to develop (Figure $5 \mathrm{~B}, \mathrm{~A}-\mathrm{A}$ plane). Secondary flow appeared unidirectional with a velocity of approximately $0.5 \mathrm{~m} / \mathrm{s}$ from the inner to the outer regions of the
U-shaped aortic arch. After entering the B-B plane of the curved aortic arch, the secondary flow was suppressed by the increasing pressure boundary condition at the outlet (Figure 5B, B-B plane). Secondary flow appeared only near the inner region of the U-shaped aorta (with the decelerated axial velocity). After passing through the U-shaped aortic arch, the strength of secondary flow gradually increased to 0.25 $\mathrm{m} / \mathrm{s}$, and contributed to the accelerated flow from the lateral side of the aorta (Figure 5B, C-C plane). In the distal descending aorta, the secondary flow diminished to two extremely weak vortices, as shown in Figure 5B, D-D plane.

At the PRFT point of the diastole, the axial velocity had a central reverse-flow jet, as opposed to the slow outer lateral forward-flow of $0.15 \mathrm{~m} / \mathrm{s}$ at the ascending aorta (Figure 5C, A-A plane). Because of the centrifugal forces, the maximal reversed flow was observed to occur in the posterior-to-anterior oval form at the curved aortic arch (Figure $5 \mathrm{C}, \mathrm{B}-\mathrm{B}$ plane). At the proximal descending aorta (Figure 5C, C-C plane), the reverse flow once again resembled that of the inner-side reversed flow. The distal descending aorta exhibited a considerably slower reverse-flow pattern (Figure 5C, D-D plane). The secondary flow pattern inside the ascending aorta at this PRF time point was in the opposite direction to that of the systolic phase; it maintained uniformity, but flowed from the outer wall into the inner aorta (Figure 5D, A-A plane). At the curvature of the aortic arch, the secondary flow exhibited counter-rotating vortices in the mid-left plane, which moved toward the posterior-outer wall (Figure 5D, B-B plane). After entering the proximal descending aorta, the secondary flow pattern disappeared, as the ascending aortic section supplied the reverse flow from the outer and lateral sides of the aorta (Figure 5D, C-C plane). The vortices of the secondary flow were observed to subside in the distal descending aortic section, as shown in the D-D plane in Figure 5D.

\section{The LD type of the DoBAR model}

The CFD simulation results of the LD type of the DoBAR model are shown in Figure 6. At the PFFT point of the systole, the primary

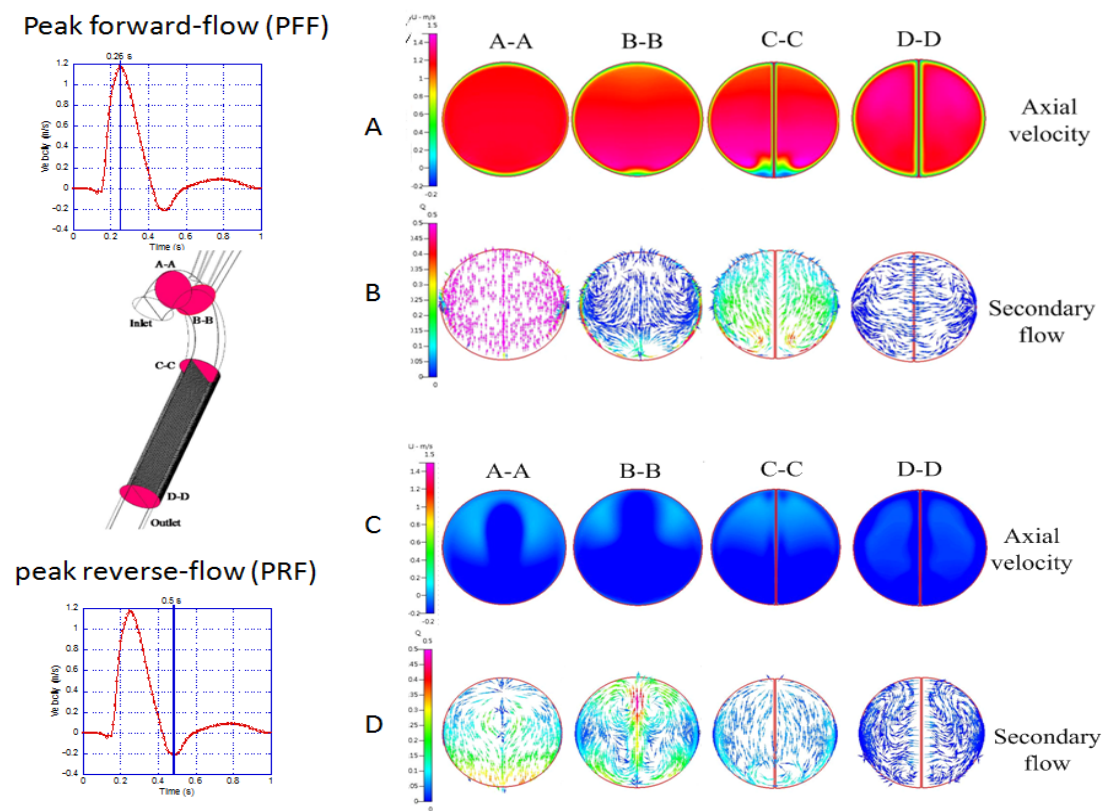

Figure 6: The CFD results of the LD type of DoBAR model. (A) Axial velocity on PFF, (B) secondary flow on PFF, (C) axial velocity on PRF, (D) secondary flow on PRF. 


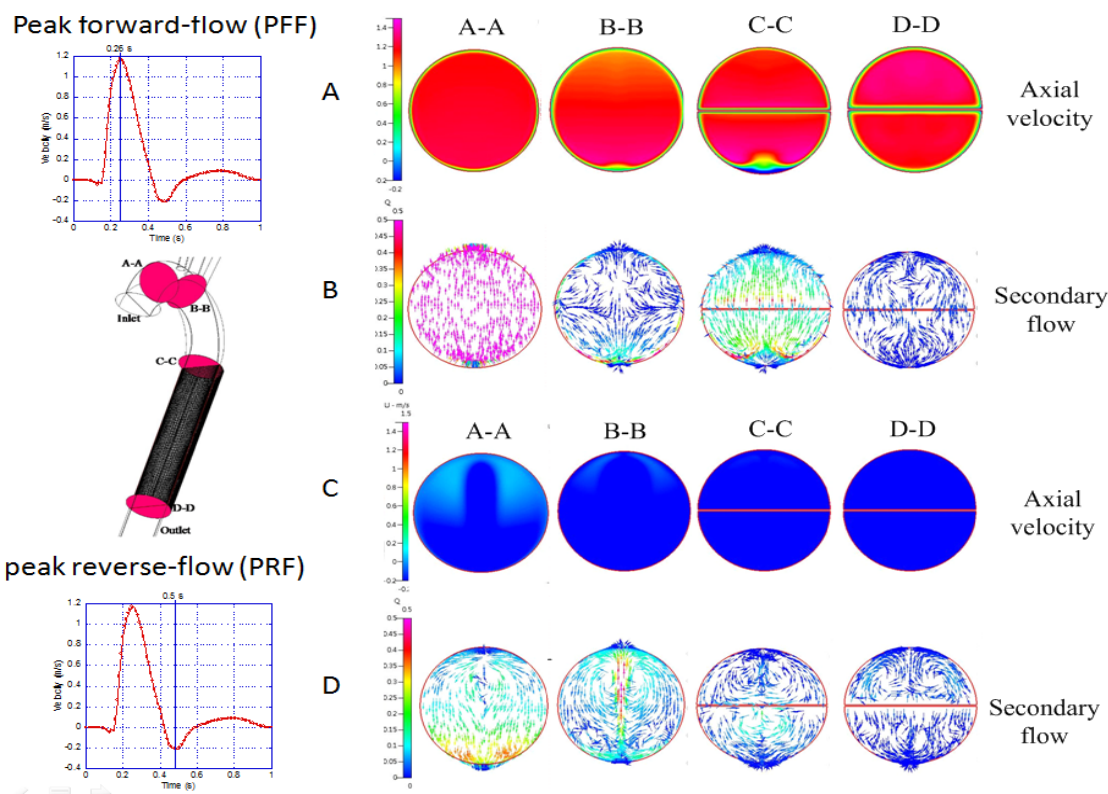

Figure 7: The CFD results of the SD type of DoBAR model. (A) Axial velocity on PFF, (B) secondary flow on PFF, (C) axial velocity on PRF, (D) secondary flow on PRF.

\begin{tabular}{|c|c|c|c|c|c|c|c|c|c|c|c|c|}
\hline \multirow[b]{2}{*}{ plane } & \multicolumn{4}{|c|}{ Strouhal number (St) } & \multicolumn{4}{|c|}{ Wormersly number $(\alpha)$} & \multicolumn{4}{|c|}{ Dean number (De) } \\
\hline & A-A & B-B & $\mathrm{C}-\mathrm{C}$ & D-D & A-A & B-B & $\mathrm{C}-\mathrm{C}$ & D-D & A-A & B-B & $\mathrm{C}-\mathrm{C}$ & D-D \\
\hline single stent-graft & 0.02 & 0.0221 & 0.0217 & 0.018 & 24.4 & 24.4 & 24.4 & 24.4 & 32.49 & 30.11 & 32.31 & NA \\
\hline LD type DoBAR & 0.0209 & 0.022 & 0.0221 & 0.022 & 24.4 & 24.4 & 24.4 & 12.2 & 33.91 & 30.07 & 31.91 & NA \\
\hline SD type DoBAR & 0.0218 & 0.0224 & 0.0242 & 0.022 & 24.4 & 24.4 & 24.4 & 12.2 & 27.46 & 27.66 & 26.28 & NA \\
\hline
\end{tabular}

Table 1: Strouhal numbers, Wormersley numbers, and Dean numbers for three models.

axial flow velocity was similar to that of the single stent-graft model at the ascending aorta (Figure 6A, A-A plane). The flow skewed and accelerated after entering the arch and the descending aorta, exhibiting the same pattern as it did with the stent-graft model (Figure 6A, B-B plane). After entering the double stent-graft region, the flow formed two mirrored separate flow patterns inside the two chambers (Figure 6A, C-C plane). In addition, the wall friction at the septum of the DoBAR hindered and slowed the axial forward-flow velocity. The forward-flow pushed against the wall on distal descending aortic region with higher outer-flow acceleration than inner-flow acceleration (Figure 6A, D-D plane). The pressure gradient in the secondary flow distribution thus changed slightly, particularly in the distal stent-graft region. The two vortices inside the aorta disappeared at the PFFT point of the systole. The two-chamber effect resulted in the secondary flow distribution, forming a bipolar mirror pattern (Figure 6B, D-D plane).

At the PRFT point of the diastole, the axial velocity exhibited the same as single stent-graft model continuous central reverse-flow jet at the ascending aorta (Figure 6C, A-A plane). Because of the effect of the distal stent-graft, the previous arch swing reverse-flow signal disappeared as the reverse flow continued to the inner-central section of the distal descending aorta (Figure 6C, B-B to D-D planes). The secondary flow pattern also lost the single-stent swing pattern at the PRFT point and, because of the dual-chamber effect, showed the bipolar mirror pattern from the arch to the descending aorta at the anterior and posterior poles (Figure 6D, B-B, C-C, and D-D planes).

\section{The SD type of the DoBAR model}

The CFD simulation results of the SD type of the DoBAR model are shown in Figure 7. At the PFFT point of the systole, the primary axial flow velocity remained the same as in the single stent-graft model at the ascending aorta (Figure 7A, A-A plane). However, because of the transverse mid-plane effects, the inner stent-graft maintained the inner flow acceleration (Figure 7A, C-C plane). Nonetheless, the outer layer of the inner stent-graft exhibited a slower flow acceleration compared with the two previous model types. The outer stent-graft also exhibited the same pattern as the inner stent-graft at the proximal descending aorta, but beyond the distal descending aorta, the outer stent-graft maintained its forward-flow acceleration (Figure 7A, D-D plane). Because of the transverse mid-plane effects, the secondary flow distribution also changed to the bipolar mirror pattern at the inner and outer poles in this systolic PFF phase (Figure 7B, B-B to D-D planes).

At the PRFT point of the diastole, the axial velocity exhibited a continuous central reverse-flow jet at the ascending aorta (Figure 7B A-A plane). Because of the effect of the distal stent-graft, the previous arch swing reverse-flow signal also disappeared, and the descending aortic region was almost entirely occupied by the reverse flow (Figure 7C, B-B to D-D planes). The secondary flow pattern also lost the singlestent swing pattern at this time point and, because of the dual-chamber effect, was substituted with the bipolar mirror pattern at the inner and outer poles from the aortic arch to the descending aorta (Figure 7D, $\mathrm{B}-\mathrm{B}$ to D-D planes). 


\section{Non-dimensional frequency parameter}

The non-dimensional frequency parameters that were needed to calculate the flow field stabilities, the Strouhal numbers, the Womersley numbers, and the Dean numbers are shown in Table 1 . The St increased slightly at the level of the aortic arch, but gradually decreased toward the distal descending aorta, with the lowest level being 0.0179 . In contrast, the St had peak values of 0.0224 and 0.0242 in the distal descending aortic level of the LD type and in the proximal descending aortic level of the SD type of the DoBAR model, respectively. The same tendency was also observed in the results of the Womersley numbers. As the flow passed via the descending aorta through the double stentgraft, the values of a decreased and reached their lowest at the level of the distal descending aorta. The Dean numbers exhibited patterns and values considerably similar to those of the single stent-graft and the LD type DoBAR model, but were lower in the SD-type DoBAR model. In the distal descending aorta (D-D plane), there was no significant centrifugal force because of the straightness of the vessel.

\section{Discussion}

Most current studies use hemodynamic measurements in the human aorta to deduce the etiological mechanisms of aortic aneurysms and aortic dissection. Several experimental and computational studies have attempted to illustrate the flow field of the aorta, to interpret the mechanisms of aortic diseases [5-7,11-13]. Qiao et al. used a simplified model of the ascending aorta and aortic arch of canines to conduct a numerical simulation analysis [12]. They demonstrated that, during the progression of the cardiac cycle, the unsteady nature of shearing stress on the walls was particularly pronounced on the inner walls of the aortic arch. Consequently, the endothelial cells in these areas were more likely to be damaged. These would then induce atherosclerosis or plaque rupturing in the intimal layer. Mori et al. created a model for CFD analysis of the specific angular distortions of the aortic arch and conducted numerical simulation analyses [13]. They observed that circumferential components contributed significantly to the hemodynamic effects on the walls of the aortic arch. Gao et al. conducted an analysis of the pulsatile blood flow between the layers of the aortic arch [14]. They found that the circumferential tension of the arterial wall is directly related to arterial pressure. These studies have validated the theory that blood flow patterns and the control of blood pressure are critical factors in the treatment of thoracic aortic aneurysm patients [5-7,11-13]. Sometimes it is difficult to obtain experimental results from humans for ethical reasons, particularly when managing aortic surgery. For this reason, the computational fluid dynamic (CFD) method has been increasingly used to identify the best treatment strategy for humans, and is a useful tool for simulating the blood flow of the human aorta [8-14]. The CFD method can provide 3D numerical simulations of blood flow through an aortic model, providing for the identification of pathological mechanisms or the development of design changes in devices used in the aorta.

This study initially used the DoBAR strategy in patients in a lifesaving capacity. However, the clinical validation of its real application, the feasibility of the DoBAR strategy, and the effect of the stent array on blood flow and the aorta need to be clarified. The CFD method is a suitable tool for this purpose. In this study, the main influence on the velocity profiles came from geometrical differences. One crucial consideration is that the effect of the double stent array on TAA cannot be predicted precisely after the stent-graft is deployed inside the aorta. Therefore, this study designed and analyzed the conditions of normal single stent-grafts and double stent-grafts in longitudinal and sagittal array scenarios. The stent-grafts were mainly located in the proximal to descending aorta. The flow at four planes was observed in these models: at the ascending aorta, the aortic arch, the proximal descending aorta, and the distal descending aorta. The axial velocity distribution and secondary flow vectors at the PFFT of the systolic phase and the PRFT of the diastolic phase were observed, respectively.

The CFD simulation results demonstrated that the primarily forward-flow pattern and its associated secondary flow at the PFFT point of the systolic phase were not significantly affected by the presence of the double stent-grafts. The only difference was that the secondary flow located in the descending aorta lost the original vortices in the single stent scenario. However, because of the effect of the double stentgrafts on the septum, the initial arch swing pattern of the reverse flow at the PRFT point of the diastole disappeared. The reverse-flow formed only a straight reversed back-flow in both the longitudinal and sagittal array scenarios of the DoBAR strategy. The secondary flow at this time point also lost the original vortex pattern and formed either upperlower or anterior-posterior bipolar secondary flow patterns.

The non-dimensional frequency parameters of the Re, $\alpha$, St, and De numbers could help identify the flow instabilities of the physiologically simulating pulsatile flow [15]. The St represents the dimensionless stroke volume, and the lower St indicates a higher pulsatility [8]. In the single stent-graft model, the St was calculated with the lowest value at the distal descending aortic segment because of the large variance between peak and mean velocity at this region in the pulsatile flow cycle. In contrast, the St had a higher average value for the DoBAR models. The Womersley numbers govern the relationship between the unsteady and viscous forces, and indicate the transition between laminar and turbulent. A Womersley parameter above 10 indicates that unsteady inertial forces are dominant, that the higher-frequency motional amplitude is reduced, and that a $90^{\circ}$ phase difference exists between the pressure gradient and the flow [5]. In the single stentgraft model, the Womersley number remained at 24 . In contrast, the Womersley numbers of the DoBAR model at the distal descending aortic level decreased because of geometrical differences between the two models. This might explain why the arch swing reverse-flow signal disappeared in the DoBAR models. However, larger Womersley numbers have a stronger influence on the flow profiles in the stenotic region [10]. Therefore, the DoBAR models tended to stabilize the flow to a greater extent than the single stent-graft models. In both DoBAR models, the Strouhal number and the Womersley number showed that the pulsatilities of the distal descending aorta segment had decreased, with a steadier flow afterward. Dean numbers can be defined as the ratio of the centrifugal inertial forces to the viscosity, and are used to assess the variance caused by the radial curvature effect along the arch of the aorta. With an increased Dean number, the centrifugal forces became stronger, thus increasing the associated secondary flow. The lowest Dean number was observed in the SD type of the DoBAR model, which means that this type had the weaker axial velocity, and thus, a weak secondary flow. These data demonstrate that the DoBAR strategy might result in a steadier flow inside the stent-grafts.

\section{Conclusion}

The DoBAR strategy for aortic repair is an alternative method for solving the specific problem of a patient with a larger aortic neck than current aortic devices can be applied to. However, the clinical validation of its real-world application needs to be examined. Using CFD simulations in three specific scenarios, the feasibility of DoBAR can be clarified. These findings will have far-reaching implications for the operation of stent-grafts in actual clinical application. The observation of blood flow and the hemodynamic changes in CFD 
Citation: Kao RH, Chen WL, Leu TS, Chen T, Kan CD (2014) Numerical Simulation of Blood Flow in Double-Barreled Cannon EVAR and its Clinical Validation. J Vasc Med Surg 2: 160. doi: 10.4172/2329-6925.1000160

models might help us better understand possible aortic flow changes and their potential effects.

\section{Acknowledgment}

This study was supported by National Science Council (NSC) grant NSC1022314-B-006-030-MY3 and National Cheng Kung University research grant NCKUH-10203025.c. In addition, this study was supported by the research of the Medical Device Innovation Center at National Cheng Kung University.

\section{References}

1. Abraha I, Romagnoli C, Montedori A, Cirocchi R (2009) Thoracic stent graft versus surgery for thoracic aneurysm. Cochrane Database Syst Rev 21.

2. Tseng E, Bush EL (2011) Thoracic Aortic Aneurysm. Medscape.

3. de Bakey ME, McCollum CH, Graham JM (1978) Surgical treatment of aneurysms of the descending thoracic aorta: long-term results in 500 patients. See comment in PubMed Commons below J Cardiovasc Surg (Torino) 19: 571 576 .

4. Wu YH, Tseng CC, Kuo TN, Yang YJ, Kan CD (2011) Double-barreled cannon stent grafts: possible solution for extremely dilated landing zone of aorta. See comment in PubMed Commons below Ann Thorac Surg 91: 1998-2000.

5. Ku DN (1997) Blood flow in arteries. Annu Rev Fluid Mech 29: 399-434.

6. Fung YC (1997) Biomechanics: Circulation (2nd ed), Springer, New York.

7. Pedley TJ (1980) The Fluid Mechanics of Large Blood Vessels, Cambridge University Press, Cambridge.
8. Fung GS, Lam SK, Cheng SW, Chow KW (2008) On stent-graft models in thoracic aortic endovascular repair: a computational investigation of the hemodynamic factors. See comment in PubMed Commons below Comput Biol Med 38: 484-489.

9. Krittian S, Oerteljr H (2010) Modelling the Human Cardiac Fluid mechanics, Proceedings of the 6th World Congress on Biomechanics, Singapore.

10. Banerjee MK, Ganguly R, Datta A (2012) Effect of pulsatile flow waveform and Womersley Number on the flow in stenosed arterial Geometry, ISRN Biomath $12: 17$.

11. Shahcheraghi N, Dwyer HA, Cheer AY, Barakat AI, Rutaganira T (2002) Unsteady and three-dimensional simulation of blood flow in the human aortic arch. See comment in PubMed Commons below J Biomech Eng 124: 378-387.

12. Qiao AK, Wu SG, Liu YJ (2001) "A finite element analysis of pulsatile flow in curved artery," J Beijing Polytech Univ 27: 240-245.

13. Mori D, Yamaguchi T (2002) Computational fluid dynamics modeling and analysis of the effect of 3-D distortion of the human aortic arch. See comment in PubMed Commons below Comput Methods Biomech Biomed Engin 5: 249260.

14. Morris L, Delassus P, Callanan A, Walsh M, Wallis F, et al. (2005) 3-D numerical simulation of blood flow through models of the human aorta. See comment in PubMed Commons below J Biomech Eng 127: 767-775.

15. Peacock J, Jones T, Tock C, Lutz R (1998) The onset of turbulence in physiological pulsatile flow in a straight tube. Exp Fluids 24: 1-9. 